\title{
Qualitative determination of occupational risks among operating room nurses
}

\author{
AUTHORS \\ ÜLFIYE ÇELIKKALP PhD' \\ AYLIN AYDIN SAYILAN PhD, RN ${ }^{2}$
}

1 Department of Public Health, Trakya University School of Medicine, Edirne, Turkey

2 Department of Nursing, Faculty of Health Sciences, Kırklareli University, Kırklareli, Turkey.

\section{CORRESPONDING AUTHOR}

ÜLFIYE ÇELIKKALP Department of Public Health, Trakya University School of Medicine, 22030, Edirne, Turkey. Phone: +90 5327225620. Email: ulfiyecelikkalp@trakya.edu.tr

\section{ABSTRACT}

Objective: The purpose of this study was to determine the occupational risks faced by operating room nurses, their working conditions and health problems, and the protective measures adopted.

Background: Since operating rooms are high-risk environments, the nurses working in them are exposed to correspondingly greater health risks.

Method: This qualitative descriptive study design involved 17 operating room nurses and was performed in a public hospital in Turkey. Data were collected by the author during face-to-face interviews using a semi-structured form constructed on the basis of the study aims. Theme establishment continued until new data emerged from the analysis of all interviews. Data were then subjected to qualitative content analysis.

Results: Three main themes were determined in the study, worker safety, working conditions, and training. At interview, operating room nurses reported being exposed to several occupational risks, including radiation, sharp implements, long working hours, and working standing up. They also reported experiencing, or were anxious about encountering in the future, various health problems associated with these risks. They also identified activities associated with education and protective measures as inadequate.
Discussion and conclusion: Several occupational risk factors in the operating room environment adversely affect the health of nurses working in the unit. We recommend that standards aimed at protecting against occupational risks be applied on a regular basis in order to preserve the personal health and safety of operating room staff.

Implication for nursing and health policy:

Nurses are responsible for the constant care of their patients under all conditions and environments but may face the risk of compromise of their own health as a result. Training, certification, and nursing policies aimed at protecting the health of employees in clinical settings should be implemented.

What is already known about the topic? Operating room nurses are known to experience severe health problems arising from their working environment. Many nurses have to cope or live with these health problems.

What this paper adds: The study reveals the need for operating room nursing education programs. It also stresses the importance of legislation and monitoring to ensure a safe working environment for nurses in Turkey.

Key words: Employee, nurse, operating room, safety. 


\section{INTRODUCTION}

Due to their different working conditions, hospitals are classified as very dangerous workplaces in terms of personnel safety under the Turkish Workplace Health and Safety Directive. ${ }^{1}$ The International Council of Nurses also drew attention to safety at work in hospitals by announcing 'Safe Environment-Safe Employment' as the theme of nurses' week in 2006 and 'Positive Practice Environments, Quality Workplaces $=$ Positive Patient Care' in $2007 .{ }^{2}$ The basic aim of health and safety at work, defined as the maintenance of workers' physical, mental and social wellbeing and raising this to the highest levels, is to protect workers from harmful workplace effects and to ensure that work is performed in a comfortable and safe environment., ${ }^{1,3}$

By the nature of their occupations, health sector workers experience occupational health problems for reasons such as exposure to various biological, chemical and ergonomic risks arising from close contact with patients, antineoplastic agents, and iodising radiation. ${ }^{13,4}$ In addition, particular concerns have been raised concerning staff health and safety due to high levels of occupational hazards faced by health staff and compensation demands. ${ }^{4,5}$ As in all workplaces, knowledge of existing or anticipated risks, and the planning and application of preventive measures must constitute the basic approach in the operating room. ${ }^{6}$ One of the most important and severe risks faced by health workers is needlestick and sharps injury (NSSI) reported at levels ranging between 23 and 68.5\% in different studies. ${ }^{13,4,7}$ Other hazards include Hepatitis HCV and HBV or Human Immunodeficiency Virus (HIV) associated with contact with patient blood or body fluids as a result of such injuries. In that context, health workers appear to be a susceptible/ vulnerable occupational group in terms of exposure to occupational diseases and associated risks. ${ }^{8}$ Essentially, all professions concerned with preserving human health and life maintain an element of stress, posing a threat to the physical and mental wellbeing of staff. However, operating room personnel have a higher risk of such injuries. ${ }^{9}$ Operating rooms are places in which advanced and complex technological equipment and tools are used, in which diverse surgical techniques and methods are applied, and which contain several potential risks to patient and personnel health. ${ }^{10,11}$ In addition, the operating room contains numerous potential risks, such as waste gasses, radiation, antiseptic and disinfectant materials, sterilisation equipment, latex allergy, trauma, psychosocial risks and burnout syndrome, needlestick or sharps injuries, infections, chemical dependency, medical waste, and fire hazards. ${ }^{1,11,12}$ Studies have described this complex nature of operating rooms and increasing workloads as significant stress factors for nurses. ${ }^{11,13}$ In addition, operating rooms are small, enclosed areas, and personnel have to work for extended periods with sharp implements and blood while making every effort to avoid errors. ${ }^{4}$ Various studies have reported an increase in sharp-tipped or - bladed object injuries, ${ }^{6}$ musculoskeletal system problems, ${ }^{14}$ and psychological problems such as burnout in operating room workers, ${ }^{15}$ in association with extended working hours and working in a standing position. Lebni et al. reported greater ergonomic and biological risks among operating room personnel, and greater lumbar disc disorder among operating room nurses. ${ }^{4}$ Another study reported that ergonomic risks played a role in increased occupational injuries among operating room staff. ${ }^{15}$ In addition, negligent use of items used in operating rooms such as lasers, radiographic equipment, and chemical sterilisers also lead to long-term health problems. ${ }^{16}$ As in all workplaces, knowledge of existing or anticipated risks, and the planning and application of preventive measures must constitute the basic approach in the operating room. ${ }^{17}$ Otherwise, unsafe/unhealthy working environments will have an adverse impact on workers' health, lower their performance, and also lead to problems with patient health. The essential step in the protection of worker health is therefore the creation of a safe and healthy work environment. There is no doubt that what workers understand by a safe and healthy working environment is one with no or little exposure to occupational risks. ${ }^{1}$ The creation of such an environment in the operating room is very difficult, although the identification of potential biological, chemical, physical, and psychosocial risks, understanding their effects, and the adoption of preventive measures are regarded as one component. ${ }^{12}$

Studies involving the operating room environment have largely concentrated on patient safety. ${ }^{18-20}$ Studies of the risks to which operating room nurses are exposed have identified work stress, workload, job satisfaction, needlestick or sharps injuries, transmission of blood and body fluids, surgical smoke, radiation, or laser hazards and musculoskeletal system symptoms, and most have focused on a single area. ${ }^{14,21,22}$

Previous studies from Turkey have been quantitative in nature, ${ }^{3,11,17}$ while studies in the international literature are quantitative and generally identify a particular risk. ${ }^{23-26}$ The present qualitative study was planned to involve all risks faced by operating room nurses by examining the working conditions and occupational exposures of these nurses in terms of personnel health, and to serve as a guide to future studies.

\section{METHOD}

\section{STUDY DESIGN AND ETHICS}

This research was designed as a qualitative descriptive study. Before the study began, written permission was obtained from the institution where the study was conducted, and ethical approval was granted by the Tekirdağ Namik Kemal University School of Medicine Non-Invasive Clinical Studies Board of Ethics (2018.141.10.06). Written and 
verbal agreement to take part was also obtained from the participants.

\section{PARTICIPANTS}

The study was performed at Kirklareli Public Hospital, Turkey, between October and December 2018. The research population consisted of nurses working in the operating room clinic $(n=35)$. The study was finally performed with 17 nurses working in the operating room for at least one year and willing to participate. The location and size of the hospital were taken into consideration in the selection of the research location.

\section{MEASUREMENTS}

A semi-structured interview form was prepared in order to identify the occupational risks faced by operating room nurses. Data were collected by an author using the in-depth interview technique at face-to-face interviews. Before data collection began, the interview form prepared by the author was first evaluated by three specialists in the field in order to establish validity and reliability. A pilot study was then carried out with five randomly selected operating room nurses who were asked to respond to open-ended questions. Following the pilot study, the requisite amendments were made to the interview form, resulting in a structured question and interview form. Interviews were held when nurses were available and felt ready for them, and in an otherwise empty room. During the interviews, data were collected from nurses with permission collected with digital voice recordings and documented. Depending on the responses given, the interviews lasted between 30 and 40 minutes.

The interview form produced a data collection tool containing 15 questions concerning nurses' descriptive characteristics (age, sex, years worked, years worked in the operating room, etc.), working conditions, occupational exposures and occupational histories. The questions, prepared in line with the existing literature concerning working conditions and occupational exposures, are given in Table 1.

\section{QUALITATIVE DATA ANALYSIS}

A qualitative content analytical approach was applied to the study data. Related concepts were collected under a specific theme. Content analysis was applied following Graneheim and Lundman, who recommended a hierarchical classification in the analysis of qualitative data. ${ }^{27}$ The steps applied in qualitative data analysis are as follows:

(1) transcription of all interviews immediately after they take place, (2) reading the entire text to understand the content, (3) arrangement and indexing of data for easy access and identification, (4) identification of meaning units and basic codes, (5) classification of the same basic codes in more comprehensive categories, (6) determining the main theme of the categories, (7) re-coding, (8) development of temporary categories, (9) the investigation of relations between categories, (10) detailing of themes, sub-themes, and categories, (11) development of the theory and the testing of that theory against the data in combination with existing information, and (12) the writing of a report containing excerpts from the original data if necessary (for example, extracts from interviews).

The transcription texts obtained by the face-to-face interview method were confirmed by operating room nurses in order to establish the validity and accuracy of the data obtained. ${ }^{27}$

\section{RESULTS}

Examination of the demographic characteristics of the 17 operating room nurses in the study revealed that almost all were women $(94.1 \%, n=16)$, and that their mean age was $39.17 \pm 8.17$ years $(\min =27, \max =53)$. Mean time worked in the operating room unit was $13.58 \pm 9.17$ years, and only four (23.5\%) had received training before joining the operating room unit.

The analysed statements were classified under three main themes: worker safety (sub-themes occupational risks, health problems, occupational accidents, and protective measures), working conditions (sub-themes, working hours, and pay), and training (sub-themes, operating room nursing certificate, in-service training).

\section{TABLE 1. INTERVIEW QUESTIONS}

Questions
Did you receive any training before starting work in the operating room unit?
How many years have you been working in the operating room unit?
Are there preventive measures aimed at protecting your health in your working environment and standards for application directives?
What are the occupational risks to which you think you are exposed in this unit?
Do you think you are developing a health problem due to working in the operating room unit? If your response is affirmative, what do you think
about the cause, and what suggestions would you make?
waste management, etc.)
Have you had an occupational accident in the operating room unit? If so, what was the cause of the occupational accident, and its outcome?




\section{TABLE 2. THEMES AND SUB-THEMES}

\begin{tabular}{|l|l|}
\hline Main Themes & Sub-themes \\
\hline Worker Safety & Occupational risks \\
\cline { 2 - 2 } & Health problems \\
\hline & Occupational accidents \\
\cline { 2 - 2 } & Protective measures \\
\hline \multirow{2}{*}{ Working Conditions } & Working hours \\
\hline \multirow{2}{*}{ Training } & Wage \\
\hline & Certificate \\
\hline & In-service training \\
\hline
\end{tabular}

\section{MAIN THEME: WORKER SAFETY}

\section{Subtheme one: Occupational risks}

The operating room nurses interviewed particularly reported such occupational risks as radiation, chemical inhalation, sharps injuries and contagious diseases in the unit, and regarded some preventive measures as inadequate. In addition, several nurses expressed concern that working in the operating room might have an adverse impact on their health.

"Permeability reports for protective equipment such as neck collars and aprons used during surgical smoke procedures that should be present in the operating room, and these should be regularly checked. However, these reports are not present in the operating room, and no information is given to us about the results of these reports. I think that this is incompatible with health and safety at work and represents a risk to our health" (Interview 2).

"We are exposed to radiation due to fluoroscopy use in orthopaedic, cardiology, and brain surgeries. However, I have never seen the permeability reports for protective equipment, barrier gloves, fluoroscopy aprons, and neck collars, and we do not use dosimetry. This sometimes worries me" (Interview 15).

"In particular, being under constant threat of biological risks due to needlestick or sharps injuries concerns me. I think that patients should be divided into risk groups in order to protect our health"(Interview 8).

\section{Subtheme two: Health problems}

Interviewees 4, 9, 14, and 17 reported an increase in lumbar pain and variceal problems after starting work in the unit and feared that they would therefore not be able to continue working in the unit much longer. In the context of this sub-theme, nurses frequently expressed concerns over their health status.

"I generally experience considerable lumbar/leg pains associated with working on foot in the same position for extended periods, and I always feel tired. In addition, the low temperature in the operating room really adversely affects our metabolism. I would prefer not to be working in this unit" (Interview 17).

"I have been working in this unit for many years, and I have lumbar and cervical hernias. I have also developed a thyroid nodule this year. I attribute this to radiation exposure. I think that such health problems would be less common if protective measures were effectively applied in our unit. I do not know how I am ever going to get my health back, and this is really upsetting me and my family" (Interview 14).

"One of the nurses in this unit developed an allergy to the highlevel disinfectant used. Everything happened so quickly that respiratory difficulty ensued and emergency intervention was necessary. We were really frightened. I am rather stressed about working in this unit. There are a great many risks here, and we may lose our health for a variety of reasons" (Interview 9).

"We become very tired since we are generally standing up all day. We frequently suffer lumbar, neck and leg pains, and these can lower our concentration. We can therefore suffer needlestick or sharps injuries. I am afraid of contracting an infectious disease" (Interview 4).

"When I became pregnant fouryears ago I requested a transfer to another department because of exposure to radiation and anesthetic gasses. But the administration turned my request down, saying there was no other suitable unit currently available. I experienced various psychological problems because of my problems with management at that time. A change of department came about following clashes with management. However, these experiences wore me down badly, and I am very concerned for my baby's health" (Interview 12).

\section{Subtheme three: Work accidents}

Approximately half (47\%) of the nurses working in the operating room reported having had at least one work accident. The most common accidents were NSSI.

"I cut my finger one day due to a careless movement while handing a scalpel to a doctor. The infection control unit was informed, and my hepatitis and ELISA test results were monitored for the next three months. I was really afraid of contracting an infectious disease" (Interview 5).

"One day a real emergency case arrived. We entered the emergency operating room, and the patient's fluids sprayed into my face and eyes as we were intervening. I washed myself with plenty of fluid for 10 minutes. I then had a blood test done, but thanks be to God, nothing came of it. We can catch any disease at any time here. Our working areas are really risky. A tiny cut can have irreversible consequences" (Interview 11).

\section{Subtheme four: Protective measures}

Nurses reiterated their opinions that the protective measures in this unit were partly insufficient and their concerns that their health was under threat. We think that this in turn led to anxiety among workers. 
"We undergo periodic health checks, but we still worry whether these are really adequate. There are a lot of risks in the operating room, such as gasses, wastes, radiation, work accidents, and working standing up. I wonder whether we can really protect ourselves with preventive measures. I would also like to state that I am very grateful to you for attracting both our own and the management's attention by listening to our problems one by one through such a study." (Interview 16).

"There are numerous risks here, and the conditions in the unit in terms of worker safety are unsatisfactory. As I said before, why are the reports concerning the protective materials we use for surgical smoke not in the operating room? We are exposed to radiation and chemical gasses, but there are no regular dosimetry checks or blood tests.” (Interview 2).

\section{MAIN THEME: WORKING CONDITIONS}

\section{Subtheme one: Working hours}

Nurses reported that there were no specified working or resting hours for the operating room, and that there were even times when they were unable to go to eat. They also emphasised that they spent these working hours in an environment that was enclosed throughout the day, without even being able to see daylight. They therefore thought that they should be regarded as a separate group from workers in other units.

"Since the operating room is a sealed area, we are not even aware what the weather is like outside. Some cases really go on for a long time, and there are times when we cannot even go and have something to eat." (Interview 7).

"Our working system involves shift work and overtime. We work all day standing up, and there are no fixed working hours set for us. We generally cannot even go for lunch. So it would be better for our working conditions to be evaluated separately." (Interview 6).

\section{Subtheme two: Salaries}

When asked what they thought about their salaries, nurses generally described themselves as unsatisfied. They described their salaries as low in the light of the workload involved.

"In my opinion, our salaries should be increased based on our workload. I think we are paid less than many other professions, given our workload. We also have to work on official holidays. We have no rest time, and we have to spend less time with our families. We receive very little additional wage for working in this unit, and our work is not reflected in our salary."

(Interview 1).

\section{MAIN THEME: TRAINING}

\section{Subtheme one: Certification}

Only one of the nurses taking part in this study held an operating room nursing certificate, and even that had been received after joining the unit. The majority of nurses started working in this area with no special training, for which reason they experienced difficulties and were even initially unaware of the occupational risks.

"I had no operating room nursing certificate when I began working in this unit. I acquired my knowledge of the operating room from colleagues already working there. However, after receiving my certificate I realised just how inadequate my knowledge was. But I am now much more confident, and it makes me happy to share what I know with colleagues." (Interview 3)

"I was given no training when I began working in this unit; but I really think that training is essential. People due to work in this unit must be aware of patient and worker safety, and comprehensive training must be provided for the establishment of minimum conditions here." (Interview 10).

"There are numerous risks here in the unit, and there is no routine working regulation. We work with numerous branches and physicians. There is a lot to know about every operation's technique, anatomy, and equipment. I think that orientation training is definitely needed." (Interview 13).

\section{Subtheme two: In-service training}

Nurses reported that in-service training programs were organised, albeit irregularly. All participants reported that training is important and requires constant repetition.

"At first I did not hold an operating room nursing certificate, and I received no orientation training. But I learned from colleagues working here in a kind of master and apprentice relationship. However, I have seen a lot of benefit from inservice training that is occasionally laid on. Training sessions are very important and must be held regularly. Otherwise, both the patient and we may come to harm." (Interview 8).

\section{DISCUSSION}

Since operating rooms are high-risk environments, the nurses working in them are exposed to correspondingly greater health risks. It is of the utmost importance to the health and safety of operating room nurses for regulations concerning their health and safety to be introduced. In the present study, and consistent with the previous literature, nurses were exposed to numerous physical (radiation etc.), biological (blood and body fluids etc.), chemical (anesthetic gasses etc.), ergonomic (excessive hours, lack of rest time), and psychological (stress, anxiety etc.) risk factors. The findings were consistent with those of various international studies, with biological agents, working conditions, and ergonomic factors representing particularly frequently reported risks. ${ }^{4,6,23}$

In contrast to previous studies, exposure to radiation was particularly considered in the present research. Nurses reported being exposed to radiation, particularly during 
fluoroscopy, that dosimetry was not employed, and that permeability reports for the protective equipment employed were not available in the operating room. Radiation exposure is particularly high in 3D scanning, which is currently highly popular and performed in the operating room (especially in $3 \mathrm{D}$ pelvic scanning at $1 \mathrm{~m}$ in height and $39.5 \mu \mathrm{Sv}$ ). This constitutes a risk for both the nurse and the patient, and it is essential that workers be given detailed information about radiation safety. ${ }^{24,28}$ The use of protective equipment and especially compliance with radiation safety are reported to be essential in order to prevent operating room personnel exposure to radiation during fluoroscopy. ${ }^{28} \mathrm{Gül}$ et al. reported that $43.2 \%$ of operating room nurses are exposed to radiation and surgical smoke more than once a day. ${ }^{11}$ They also reported that no radiation hazard signs were present in approximately half of operating rooms, and that $70.5 \%$ of nurses were unaware how functional the protective equipment was. ${ }^{11}$ In addition, the equipment used for protection against radiation must be checked at least once a year for tears, holes, and thinning, and it is essential for personnel to use dosimetry. ${ }^{29,30}$ Our findings are consistent with those of other studies, and show that radiation is widely employed and that despite its known deleterious effects, sufficient protective measures are not taken against it. In addition, a finding of inadequate protective measures against exposure to radiation further justifies the participants concern about their safety.

Another important risk factor reported by operating room nurses in this study involves accidents arising from NSSIs and associated contact with blood and body fluids. Different studies have reported greater NSSI among operating room, emergency department, and intensive care unit nurses. ${ }^{22,25}$ However, the causes of these accidents are generally related to the working environment. ${ }^{1}$ One study reported that the rate of needle injuries increased by $16 \%$ with every 10 hours worked. ${ }^{26}$ Another study involving 2,031 operating room nurses from 247 hospitals in Thailand reported longer weekly working hours, personnel shortages, fatigue, starting work without appropriate training, associated lack of awareness of the dangers in the working environment, and failure to use personal protective equipment among the causes of contact with blood and body fluids. That study also reported a greater incidence of NSSI among nurses working for extended periods on foot and working overtime. ${ }^{21}$ Our findings that nurses generally worked shifts and overtime, and spent all day working standing up are consistent with the previous literature. Our results show that nurses, despite all their intensive work and labour, are nevertheless exposed to occupational risks because of their working environment.

One of the most important findings of this study involves operating room nurses' anxieties concerning severe health problems or occupational disease due to exposure to any occupational risk. Several of the nurses in this study reported having various health problems because of their work (such as musculoskeletal diseases, allergies, needlestick or sharps injuries, infections, and psychological problems) or else feared that they might contract a health problem or occupational disease at any time. For example, one participant reported a needlestick injury in one emergency case and feared catching a disease at any moment. In a study from Iran, Fathi et al. noted that a greater incidence of sharp-edged object injuries in operating room workers was an expected finding. ${ }^{23}$ Another study of operating room personnel in Egypt emphasised that insufficiently applied safe injection procedures lead to a greater prevalence of NSSI. ${ }^{6}$ One study of health workers infected with hepatitis $B$ reported that $37 \%$ of participants had developed that infection following NSSI or contact with risky material. ${ }^{11}$ We think that a high risk of transmission and inadequate safety precautions increase operating room nurses' anxieties about contracting a health problem. Foda et al. reported a greater incidence of needlestick injuries among operating room nurses with long working hours and lacking experience and training. ${ }^{6}$ Both the Centers for Disease Control and Prevention (CDC) and the World Health Organization (WHO) have reported the need for safe injection procedures to be adapted for operating rooms in order to protect against needlestick injuries. Consistent with the present research, previous studies have found that safe injection procedures are not adequately applied. ${ }^{4,6}$ We think that the insecurity caused by the high risk of transmission in both the present and other studies, and the lack of standardisation in terms of actions to be taken following transmission (reporting, immunisation, and monitoring) cause increased anxiety over the contraction of health problems among operating room nurses. These concerns among health workers about the increasing risk of transmission will inevitably worsen with the COVID-19 pandemic. Health workers on the front line have been the occupational group with the highest risk of contagion during the COVID-19 pandemic. The risk of COVID-19 infection, which is particularly transmitted by respiratory secretions, is reported to be higher for nurses in the operating room, an area with particularly infectious characteristics. $^{32}$

Operating room nurses in this study also complained of lower back/foot pains. Studies of musculoskeletal system disorders in nurses have observed a higher prevalence of lumbar hernia in nurses than in other health workers. ${ }^{22,33}$ Aydın et al. determined musculoskeletal system problems in as many as $84 \%$ of operating room nurses, and emphasised that these were at risk in terms of body posture. ${ }^{14}$ Although working environment risks are inevitable, health problems can be reduced if protective measures are applied appropriately and regularly in line with the standards set out by law and in the relevant directives.

Factors such as occupational risks, working standing up, excessive hours, and insufficient rest time are reported to increase nurses' stress levels and workload and to reduce their job satisfaction levels. ${ }^{10,34}$ In the present study, nurses 
reported being unable to go for lunch because of their workloads, to be unable to set aside sufficient time for their families, and therefore being discontented with their working hours, and even being unwilling to work in the unit any longer. One cross-sectional descriptive study of 96 operating room nurses in Finland reported that operating rooms are stressful and complex environments, that a workplace culture needs to be established within the unit, and that nurses' stress levels are directly correlated with their job satisfaction. That study also identified greater workload stress in operating room nurses. ${ }^{10}$ Several different studies have similarly emphasised that factors such as adverse physical environmental conditions, inadequate materials and personnel, workload, and work stress all increase burnout levels. ${ }^{22,34-36}$ Additionally, the lack of a satisfactory salary reflecting the service provided also has an adverse impact on job satisfaction.

One important point particularly noted by one operating nurse in this study concerned working during pregnancy. That participant reported being unwilling to work in the operating room during pregnancy for reasons of fetal health and having requested to work in a different unit, although this had initially been declined. Although the majority of nurses are women, studies concerning pregnancy are limited. ${ }^{37-39}$ Studies of the occupational risks faced by pregnant midwives and nurses have reported that ward changes are necessary in order to minimise teratogenic and fetotoxic risks and hazardous conditions in units such as oncology, radiology, and the operating room. ${ }^{37-39}$ Studies have also reported that chemicals such as anaesthetic gasses and drugs, and cases of pregestational infertility, spontaneous abortus, preterm birth, and low birth weight babies are more common in the operating room. ${ }^{1,39}$ In addition, greater spontaneous abortus has been reported in nurses who work extended periods working standing up and overtime. 37,38 Pregnancy is a uniquely special time in a woman's life and must be considered from the perspective of the health of the next generation. We think that our findings show that policies for protecting hospital worker health are inadequate.

International Labor Organization (ILO) Nursing Personnel Convention 149 concerning staff working conditions and advisory recommendation No 157 advise a 40-hour working week, a working day of 8-12 hours, a weekly uninterrupted rest period of 36-48 hours, and an uninterrupted period of at least 12 hours between shifts. ${ }^{\circ}$ However, studies from Turkey, which is not one of the 38 signatory countries, report that nurses work 16-24 h shifts and more than 45 h a week. ${ }^{22,37}$ We think that excessive working hours, which may be attributed to personnel shortages, can only be rectified with the employment of sufficient numbers of qualified staff.

Our research showed that unit-specific health screening was not performed regularly/routinely. Yet ILO Convention No. 149 emphasises the importance of regular health examinations for health workers and of protecting personnel health. Our finding identifies a significant obstacle to early diagnosis and treatment.

The operating nurses interviewed in the present study stated that they held no operating room nursing certificates before joining the unit, and most also received no orientation training. We also determined that in-service training programs were not held regularly. Some nurses in this study also possessed insufficient knowledge when they began working in the unit, and in-house training programs were not regularly arranged. Nurses also reported that their knowledge levels increased after instruction, and that education was of very great importance. Yet the Turkish Ministry of Health In-Service Training Decree (No. 185 dated 2009) and the Occupational Safety and Health Administration Recommended Practices for Safety and Health Programs Education and Training (OSHA, 2016) both explicitly state that newly beginning and existing personnel should both receive training concerning occupational risks and protective measures. ${ }^{41,42}$ Receipt of appropriate training and registration with the Nursing and Midwifery Council in the UK, or equivalent institutions in other countries, is required to become an operating room nurse in Europe, but no special education is required in Turkey. Although there is a 96-hour operating room nursing course in Turkey, the fact that it is not essential to have completed this to work in the operating room is a major educational deficiency. However, since various different types of surgeries are performed in operating rooms, staff need to be given new and updated information. Studies confirm that operations are constantly being modified by new technological methods, for which reason, information must constantly be updated ${ }^{4,23,43}$, and that refresher training supported by simulations, video techniques, robotics and teletype applications is essential in order to increase the professional sufficiency of such education. ${ }^{23,43-45}$ Tanaka et al. described a lack of sufficient knowledge in the operating team as an important source of conflict. ${ }^{46}$ Operating room nurses in Sweden reported that they required complementary information in the preoperative period in addition to their initial training, and that if they were not properly informed concerning procedures and applications it was impossible to prepare themselves properly, for which reason refresher training was essential. ${ }^{43}$ Different studies have described the emphasised training sessions in particular as of the greatest importance to personnel health and safety, and that these need to be provided at each stage (before starting work, during work, after an occupational accident, and periodically). It must not be forgotten that health workers may encounter a potential risk of any kind at any moment. The COVID-19 pandemic being experienced across the world has once again shown the importance of education and awareness if personnel are to protect themselves, and that health problems will occur if the requisite precautions are not adopted. Current health problems and protective measures should now be added to training and education. 


\section{CONCLUSION}

This qualitative study performed to determine the occupational risks facing operating room nurses showed that nurses were particularly exposed to radiation, NSSI, and biological and ergonomic risks, and that they experienced a range of health problems. We also determined that protective measures and occupation training against occupational risks were not provided regularly. Risk factors were identified in this study, but no analysis was made concerning which factor resulted in greater risk. More extensive studies with larger samples may be recommended to determine risk coefficients among factors and to permit generalisation.

Although there are several standards aimed at protecting the health and safety of all workers, in Turkey and internationally, there are no special practice standards for operating rooms. It is of great importance in terms of protecting worker health for both domestic regulations and ILO recommendations regarding occupational risks, protective measures, working hours, salaries, etc. to be adopted and applied. Operating room nurses are at serious risk. Administrators must therefore monitor whether or not protective measure standards are being applied. Moreover, health personnel must be given continuous training regarding the operating room if worker health is to be protected. Personnel training and perioperative care procedures need to be updated in light of new health problems as these are identified.

Nurses must be provided with material and psychological support in order to reduce the occupational risks, heavy workload, and work stress levels and to improve the working conditions of operating room nurses.

\section{LIMITATIONS OF STUDY}

The principal limitation of this study is that since it was performed with operating nurses in a single public hospital, the results cannot be generalised. Individual interviews allowed us to obtain in-depth insights into the different reported factors and their interactions but did not provide a broad picture. The interview method does not allow the sharing and comparison of perceptions that occurs during the interactions typical of focus groups.

\section{CONFLICTS OF INTEREST}

The authors declare that there are no conflicts of interest.

\section{REFERENCES}

1 Bilir B. Occupational Health and Safety. Güneș Tıp Kitapevleri. Ankara. 2016

2 International Council of Nurses (ICN). Positive Practice Environments: Quality Workplaces = Quality Patient Care: Information and Action Tool Kit. [cited 2019 Apr 4] Available from: http://www.icn.ch/images/stories/documents/ publications/ind/indkit2007.pdf

3 Çelikkalp Ü, Saraçoğlu GV, Keloğlu G, Bilgiç Ș. The assessment of work places of nurses on occupational safety [Turkish] TAF Prev Med Bull. 2016; 15(5): 408-13.

4 Lebni JY, Azar FA, Sharma M, Zangeneh A, Kianipour N, Azizi SA, et al. Factors affecting occupational hazards among operating room personnel at hospitals affiliated in Western Iran: a crosssectional study. J Public Health (Berl.). 2020; [in press]

5 Ndejjo R, Musinguzi G, Xiaozhong Y, Esther B, Musoke D, Wang JS, Halage AA, Whalen C, Bazeyo W, Williams P, Ssempebwa J. Occupational Health Hazards among Healthcare Workers in Kampala, Uganda. J Environ Public Health. 2015; 1-9.

6 Tawfik Foda NM, Mohamed Elshaer NS, Mohamed Sultan YS. Safe injection procedures, injection practices, and needlestick injuries among health care workers in operating rooms. Alexandria J Med. 2018; 54(1): 85-92.

7 Çelikkalp Ü, Dilek F. Factors affecting the occupational accident rates among nurses. Rev Esc Enferm USP. 2019; 53: e03524.

8 Al-aslami RA, Elshamy FM, Maamar EM, Shannaq AY, Dallak AE, Alroduni AA. Knowledge and awareness towards occupational hazards and preventive measures among students and dentists in Jazan dental college, Saudi Arabia. Open Access Maced J Med Sci. 2018; 6(9): 1722-26.

9 Edmund E. Analysis of occupational hazards and safety of workers in selected working environments within Enugu metropolis. J Environ Analyt Toxicol. 2015; 5(6): 1-6.

10 Eskola S, Roos M, McCormack B, Slater P, Hahtela N, Suominen T. Workplace culture among operating room nurses. J Nurs Manag. 2016; 24(6): 725-34.

11 Gül A, Andsoy, I, Görücü R, Özen B. Examination of operating staff's practice about scopy use and safety [Turkish]. Balikesir Health Sciences Journal. 2019; 8(1): 1-6.

12 Eti Aslan, Kan Öntürk F. Safe operating room environment; biological, chemical, physical and psychosocial risks, effects and precautions [Turkish]. Maltepe Üniversitesi Hemșirelik Bilim ve Sanatı Dergisi. 2011; 4(11): 133-40.

13 Önder G, Aybas M, Önder E. Determining the precedence order of the factors influencing nurses' stress level using multi criteria decision making techniques. Optimum Ekonomi ve Yönetim Bilimleri Dergisi. 2014; 1(1): 1-15.

14 Aydın Sayılan A, Öztekin SD. Operating room nurses' working posture and its predictors in hospital [Turkish]. Gümüșhane University Journal of Health Sciences, 2018; 7(1): 23.

15 Mahmoudifar $Y$, Seyedamini B. Ergonomic relationship during work in nursing staff of intensive care unit with operating room. Int Arch Health Sci. 2017; 4(2): 42-7.

16 Lin LY, Juan CW. A systematic review of the literature: risk factors and strategies for occupational hazards in hospitals. Chinese J Occup Med. 2015; 22(2): 71-88.

17 Andsoy I. Topic that must be known by surgical team: what are the risks of fire in operating rooms? How to establish fire security [Turkish]. TAF Prev Med Bull. 2013; 12(4): 449-54. 
18 Kapıkıran G, Bülbüloğlu S, Eti Aslan F. Patient safety, patient safety culture, medical errors and adverse events in the operating room. Journal of Health and Nursing Management. 2018; 5(2): 132-40.

19 Jung JJ, Elfassy J, Jüni P, Grantcharov T. Adverse events in the operating room: definitions, prevalence, and characteristics. a systematic review. World J Surg. 2019; 43(396): 1-14.

20 Schwendimann R, Blatter C, Lüthy M, Mohr G, Girard T, Batzer $\mathrm{S}$, et al. Adherence to the WHO surgical safety checklist: an observational study in a Swiss academic center. Patient Saf Surg. 2019; 13(14): 1-6.

21 Kasatpibal N, Whitney JD, Katechanok S, Ngamsakulrat S, Malairungsakul B, Sirikulsathean $P$, et al. Practices and impacts post-exposure to blood and body fluid in operating room nurses: a cross-sectional study. Int J Nurs Stud. 2016; 57: 39-47.

22 Uğurlu Z, Karahan A, Ünlü H, Abbasoğlu A, Özhan Elbaș N, Avcı Ișik S, et al. The effects of workload and working conditions on operating room nurses and technicians. Workplace Health Saf. 2015; 63(9): 399-407.

23 Fathi Y, Barati M, Zandiyeh M, Bashirian S. Prediction of preventive behaviors of the needlestick injuries during surgery among operating room personnel: application of the health belief model. Int J Occup Environ Med. 2017; 8(4): 232-40.

24 Querido FM, De Brito Poveda V. Exposure of nursing staff to radiation in the operating room: a descriptive study. Rev Sobecc Sao Paulo. 2015; 20(1): 2-8.

25 Chiou ST, Chiang N, Wu CH, Chien LY. Health issues among nurses in Taiwanese hospitals; national survey. Int J Nurs Stud. 2013; 50(10): 1377-84.

26 Clark SP. Hospital work environments, nurse characteristics, and sharps injuries. Am J Infect Control. 2007; 35(5): 302-9.

27 Graneheim UH, Lundman B. Qualitative content analysis in nursing research: concepts, procedures and measures to achieve trustworthiness. Nurs Educ Today. 2004; 24(2): 105-12.

28 Schuetze K, Kraus M, Eickhoff A, Gebhard F, Richter PH. Radiation exposure for intraoperative 3D scans in a hybrid operating room: how to reduce radiation exposure for the surgical team. Int J Comput Assist Radiol Surg. 2018; 13(8): 1291-300

29 Kaplan DJ, Patel JN, Liporace FA, Yoon RS. Intraoperative radiation safety in orthopaedics: a review of the ALARA (as low as reasonably achievable) principle. Patient Saf Surg. 2016; 10: 27.

30 Gökharman FD, Aydın S, Koșar PN. Need to know about radiation safety vocationally. Sağlık Bilimleri Dergisi. 2016; 7(2): 35-40.

31 Deisamhammer S, Radon K, Nowak D, Reichert J. Needlestick injuries during medical training. J Hosp Infect. 2006; 63(3): 263-67.

32 Chen X, Shang Y, Yao S, Liu R, Liu H. Perioperative care provider's considerations in managing patients with the COVID-19 Infections. Transl Perioper Pain Med. 2020; 7(2): 216-24.

33 Ribeiro T, Serranheira F, Loureiro H. Work related musculoskeletal disorders in primary health care nurses. Appl Nurs Res. 2017; 33: 72-7.

34 Atefi N, Abdullah KL, Wong LP. Job satisfaction of Malaysian registered nurses: a qualitative study. Nurse Crit Care. 2014; 21(1): 8-17.

35 Martin DM. Nurse fatigue and shift length: a pilot study. Nurs Econ. 2015; 33(2): 81-7.
36 Öcal D, Kürklu S, Tekin K. Determining the stress and motivation levels of the nurses working at the surgery clinics of a training and research hospital. Health Care Acad J. 2015; 2(3): 147-54.

37 Çelikkalp Ü, Yorulmaz F. The effect of occupational risk factors on pregnancy and newborn infants of pregnant midwives and nurses in Turkey: a prospective Study. Int J Caring Sci. 2017; 10(2): 690-703.

38 Lawson CC, Rocheleau CM, Whelan EA, Hibert EN, Grajewski B, Spiegelman $D$, et al. Occupational factors and risk of preterm birth in nurses. Am J Obstet Gynecol. 2009; 200(1): 51-8.

39 Lawson CC, Rocheleau CM, Whelan EA, Lividoti Hibert EN, Grajewski B, Spiegelman D, et al. Occupational exposures among nurses and risk of spontaneous abortion. Am J Obstet Gynecol. 2012; 206(4): 327.e.1-8.

40 International Labour Office (ILO), ILO Nursing Personnel Convention No.149, Recognize their contribution Address their needs. 2005. [cited 2019 May 5] Available from: https://www. ilo.org/wcmsp5/groups/public/---ed dialogue/---sector/ documents/publication/wcms 508335.pdf

41 Ministry of Health In-Service Training Regulation, Turkey [Turkish]. 2009. [cited 2019 May 21] Available from: https://kms. kaysis.gov.tr/Home/Goster/60244

42 Occupational Safety and Health Administration (OSHA). Recommended practices for safety and health programs. education and training. 2016. [cited 2019 May 21] Available from: https://www.osha.gov/shpguidelines/education-training. $\underline{\text { html }}$

43 Sandelin A, Kalman S, Gustafsson BA. Prerequisites for safe intraoperative nursing care and teamwork: operating theatre nurses' perspectives: a qualitative interview study. Clin Nurs. 2019; 28: 2635-43.

44 Zhaoa X, Cong L. Effect of problem and scripting-based learning combining wearable technology on orthopedic operating room nurses' learning outcomes. Nurse Educ Today. 2019; 73: 13-6.

45 Park EW, Lee H, Yun EK. Development and evaluation of a quick response code-based nursing education program for operating and recovery room nurses. Comput Inform Nurs. 2019; 37(11): 599-605.

46 Tanaka P, Hasan N, Tseng A, Tran C, Macario A, Harris I. Assessing the workplace culture and learning climate in the inpatient operating room suite at an academic medical center. J Surg Educ. 2019; 76(3): 644-51. 